Document downloaded from:

http://hdl.handle.net/10251/91909

This paper must be cited as:

Page Del Pozo, AF.; De Rosario Martínez, H.; Mata Amela, V.; Besa Gonzálvez, AJ. (2014). Model of Soft Tissue Artifact Propagation to Joint Angles in Human Movement Analysis. Journal of Biomechanical Engineering. 136:1-7. doi:10.1115/1.4026226

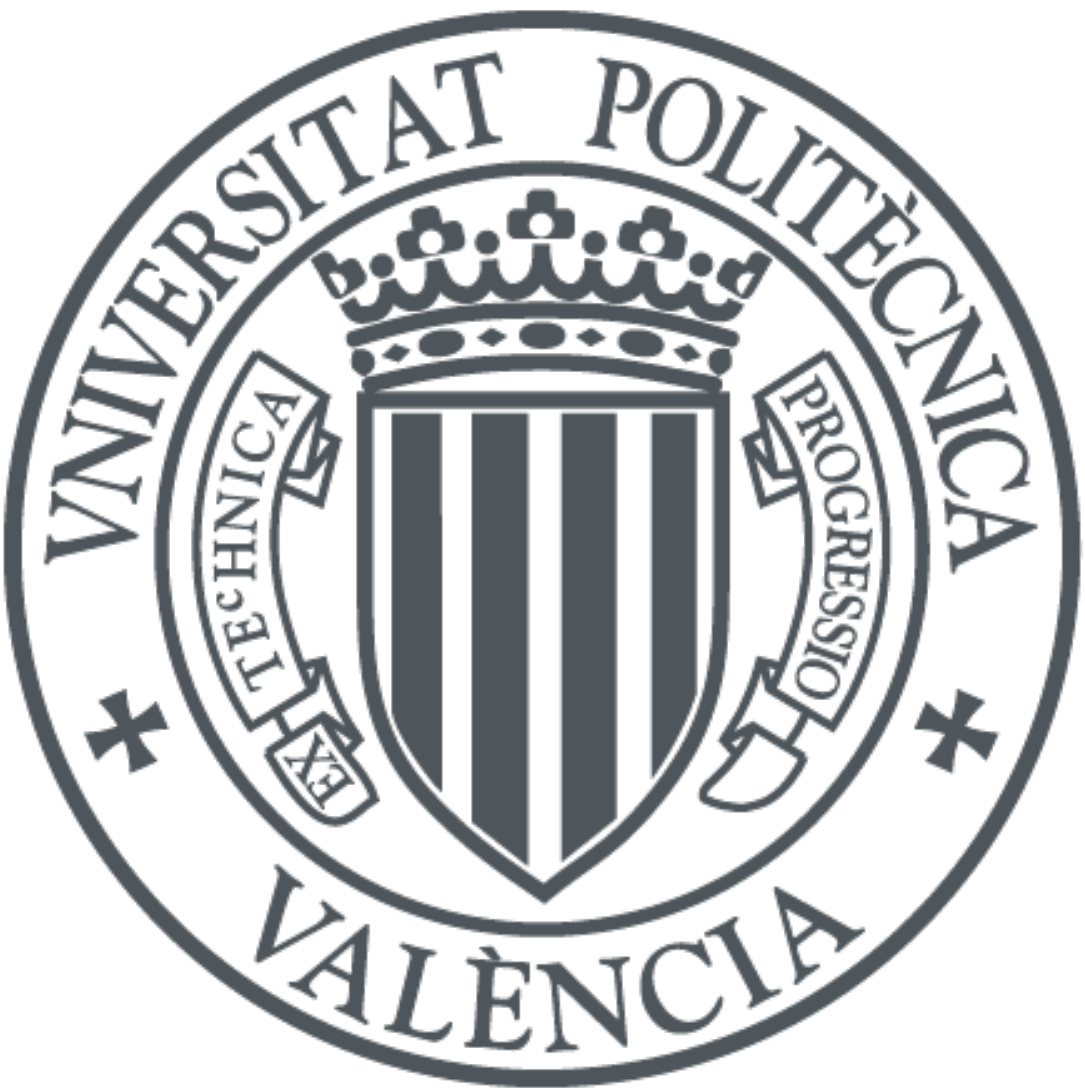

The final publication is available at

http://dx.doi.org/10.1115/1.4026226

Copyright ASME International

Additional Information 


\section{Model of soft tissue artifact propagation to joint angles in human movement}

analysis.

Álvaro Page ${ }^{(1,4)}$, Helios de Rosario ${ }^{(2,4)}$, Vicente Mata ${ }^{(3)}$, Antonio Besa $^{(3)}$

${ }^{(1)}$ Dept. Física Aplicada. Universidad Politécnica de Valencia. Camino de Vera s/n, 46022

VALENCIA (SPAIN). Tel: +34 963877528. Fax: +34 963877528.

${ }^{(2)}$ Instituto de Biomecánica de Valencia. Universidad Politécnica de Valencia. Camino de Vera s/n, 46022 VALENCIA (SPAIN). Tel: +34 963879160. Fax: +34 963879169.

${ }^{(3)}$ Departamento de Ingeniería Mecánica y de Materiales. Universidad Politécnica de Valencia.

${ }^{(4)}$ Grupo de Tecnología Sanitaria del IBV, CIBER de Bioingeniería, Biomateriales y Nanomedicina (CIBER-BBN).

\section{ABSTRACT}

This work describes the kinematic laws that govern the transmission of soft tissue artifact errors to kinematic variables in the analysis of human movements. Artifacts are described as relative translations and rotations of the marker cluster over the bone, and a set of explicit expressions is defined to account for the effect of that relative motion on different representations of rotations: the rotation around the screw axis, or rotation vector, and the three Euler angle sequences that are most frequently used in the field of biomechanics (XY'Z', YX'Y', ZX'Y', ). Although the error transmission is nonlinear in all cases, the effect of artifacts is greater on Euler sequences than on the rotation vector. Specifically, there are crosstalk effects in Euler sequences that amplify the errors near singular configurations. This fact is an additional source of variability in studies that describe artifacts by comparing the Euler angles obtained from skin markers, with the angles of an artifactfree gold standard. The transmission of errors to rotation vector coordinates is less variable or dependent on the type of motion. This model has been tested in an experiment with a deformable mechanical model with a spherical joint.

Keywords: Human movement analysis, Stereophotogrammetry, Soft tissue artifacts, assessment 


\section{Introduction}

Soft tissue artifacts (STA) are the main source of errors in human movement analysis by videophotogrammetry [1]. There are many published studies that describe STA and propose strategies to minimize their effects [2]. However, the facts revealed by those studies are partly obscured by the variety of conventions for representing STA, and how they affect the nature of transmitted errors.

STA involve a relative movement of the marker cluster over the underlying bone. They are usually represented as the difference between measured joing angles and a "gold standard" obtained by a STA-free technique [3-7]. However, that measure may be different from the actual relative movement, since the compositions of rotations is strongly nonlinear [8].

The mathematical representation of rotations may also affect the nature of STA errors. Euler angles (EA) are probably the most popular way of expressing human joint rotations, although they can present singularities [9]. It has also been shown that EA may cause large amplifications of instrumental errors and artifacts [10,11] . Other studies use rotation or attitude vectors $(\mathrm{RV}$, henceforth represented by $\boldsymbol{\theta}=\theta \mathbf{u})$, which Woltring proposed as a convenient way for representing joint rotations [10]. Closely related is the Rodrigues vector (represented by $\mathbf{\Omega}=\tan (\theta / 2) \mathbf{u},[12]$ ), a scaled version of the RV that can be obtained from marker coordinates with linear operations [8]. This representation has the advantage of not having singularities, but its meaning is less intuitive in terms of anatomically-defined axes, and this may be a reason why they are less reported than EA in biomechanical studies.

This paper presents the equations that quantify the error transmitted by STA to kinematic variables. Artifacts are described as the relative motion between marker 
clusters and the underlying bones. Their effect is evaluated depending on how rotations are expressed: either by RV or by three EA sequences that are commonplace in the field of biomechanics: $X Y^{\prime} Z^{\prime \prime}$, which is used in knee in gait analysis [13], $Z Y^{\prime} X^{\prime \prime}$, for spine and lower or upper limbs [14,15], and $Y^{\prime} Y^{\prime \prime}$, which is recommended for the gleno-humeral joint [15]. These rules are validated by means of an experiment with a mechanical analogue, that simulates a body segment with a controlled motion.

\section{Kinematic model}

STA are described as a relative motion between the area of skin where markers are attached and the underlying bone. This motion may be assumed to be a small rotation, $d \mathbf{q}$, combined with a translation of the marker cluster with respect to its reference

position, $\mathbf{G}_{0} \mathbf{G}_{2}$. Experimental measures of $\left\{\mathrm{d} \boldsymbol{\theta} ; \mathbf{G}_{0} \mathbf{G}_{2}\right\}$ can be obtained as described in [16], by the following steps (Fig. 1):

- Compute the real motion of the bone from a reference position $\mathrm{B}_{0}$ to the pose at time t, B. This motion is defined by the RV $\boldsymbol{\theta}$ or by the Euler sequence $\left\{\Phi_{1}, \Phi_{2}, \Phi_{3}\right\}$, plus the translation $\mathbf{G}_{0} \mathbf{G}_{1}$ of an arbitrary point.

- Measure the location of markers attached to the skin at the reference position $\mathrm{MC}_{0}$ (points $\mathrm{P}_{\mathrm{i} 0}$ ) as well as at position $\mathrm{MC}$ at time $\mathrm{t}$ (points $\mathrm{P}_{\mathrm{i}}$ ). This motion can be described as a rotation $\boldsymbol{\theta}_{M C}$ or $\left\{\Phi_{M C 1}, \Phi_{M C 2}, \Phi_{M C 3}\right\}$, plus the translation $\mathbf{G}_{0} \mathbf{G}$, and it is the observed movement with artifact.

- Move the markers $\mathrm{P}_{\mathrm{i}}$ back to $\mathrm{P}_{\mathrm{i} 2}$ by applying the inverse displacement $\left\{-\boldsymbol{\theta} ;-\mathbf{G}_{0} \mathbf{G}_{1}\right\}$. Then MC moves to $\mathrm{MC}_{2}$ (dotted outline in Fig. 1). The artifact 
$\left\{\mathrm{d} \boldsymbol{\theta} ; \mathbf{G}_{0} \mathbf{G}_{2}\right\}$, in a bone-embedded reference system, is the rigid displacement between $\mathrm{MC}_{0}$ and $\mathrm{MC}_{2}$.

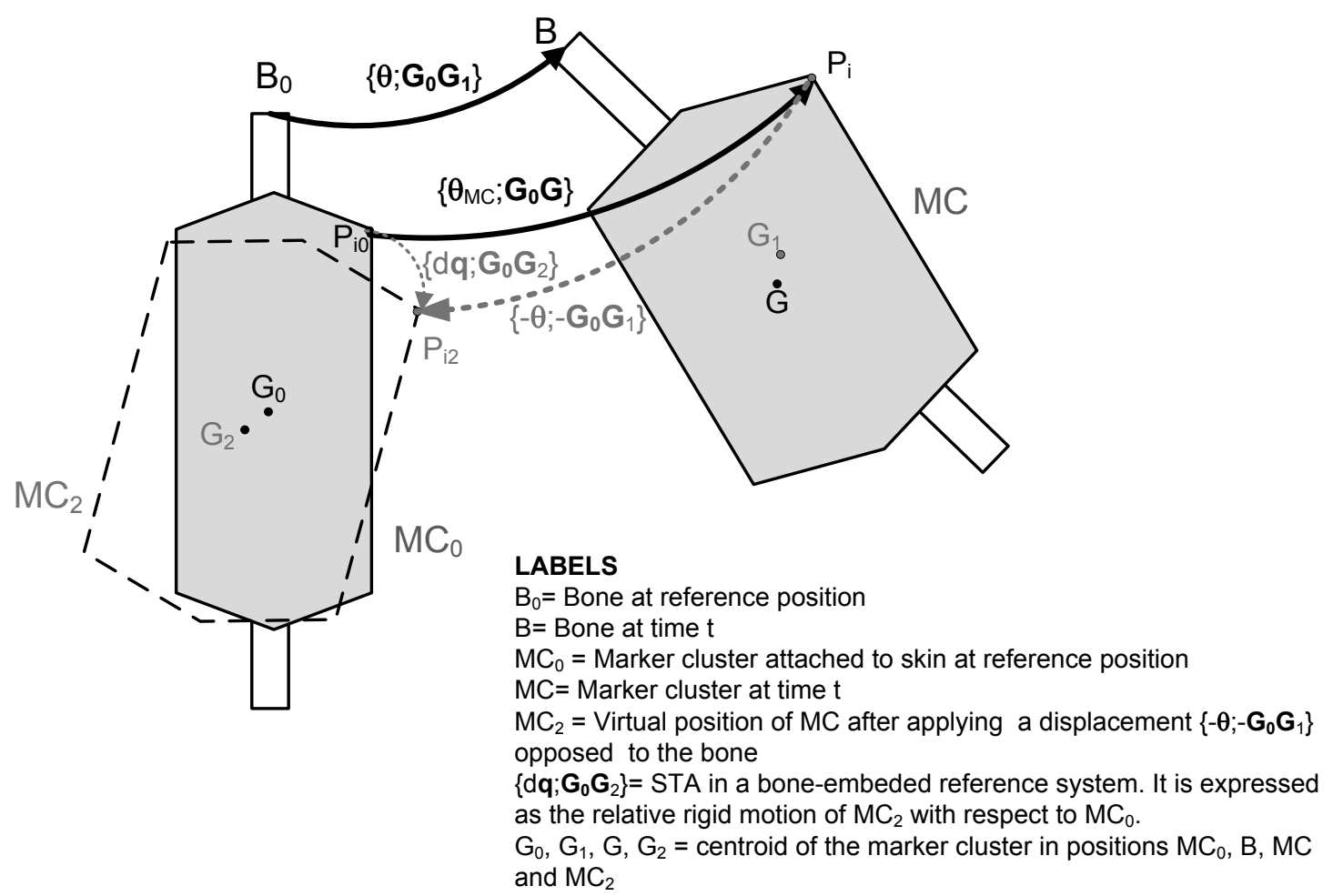

Figure $1 . \mathrm{B}_{0}$ represents the location of bone in the reference position. It moves to a given position $\mathrm{B},\left\{\boldsymbol{\theta} ; \mathbf{G}_{0} \mathbf{G}_{1}\right\}$ being the associated finite displacement. $\mathrm{MC}_{0}$ represents the cluster of markers attached to the skin, at reference position (points $P_{i 0}$ ). When $B_{0}$ moves to $\mathrm{B}, \mathrm{MC}_{0}$ goes to $\mathrm{MC}\left(\right.$ points $P_{i}$ ) by means of the finite displacement $\left\{\boldsymbol{\theta}_{M C} ; \mathbf{G}_{0} \mathbf{G}\right\}$. STA are expressed in a bone-embedded reference frame by the displacements $\boldsymbol{P}_{i 0} \boldsymbol{P}_{i 2}$ of the individual markers with respect to the bone. $P_{i 2}$ are obtained from the observed positions of the markers at $M C, P_{i}$, after applying the finite displacement $\left\{-\boldsymbol{\theta} ;-\boldsymbol{G}_{0} \boldsymbol{G}_{1}\right\}$, opposite to the real motion of the bone. The associated rotation $\mathbf{q}$ represents the relative rotation of the whole cluster with respect to the bone. 
Note that the displacement from $\mathrm{MC}_{0}$ to $\mathrm{MC}$ involves both rigid motion as well as some deformation, but due to rigid motion constraints, the deformation component does not affect the results of usual motion analysis procedures [16].

The difference between the "real" and "observed" translations $\left(\mathbf{G}_{0} \mathbf{G}_{1}\right.$ and $\left.\mathbf{G}_{\mathbf{0}} \mathbf{G}_{2}\right)$ has the same size as the translation artifact $\left(\mathbf{G}_{0} \mathbf{G}_{2}\right)$. However, the difference between the rotations (be them represented by RV, EA, or any other formalism) may be very different from $d \mathbf{q}$, and it is very dependent on the way rotations are represented, as described in the following sections.

\section{Transmission to the rotation vector}

The error transmitted by the rotation artifact $d \mathbf{q}$ to the RV is the vector difference:

$$
d \boldsymbol{\theta}=\boldsymbol{\theta}_{M C}-\boldsymbol{\theta}
$$

This vector can be defined by its module $(d \theta)$ and direction $(d \mathbf{u})$. If the error is small, a linear approximation to $d \boldsymbol{\theta}$ may be sufficiently accurate:

$$
d \boldsymbol{\theta}=d \theta \mathbf{u}+\theta d \mathbf{u}
$$

It is also possible to express $d \boldsymbol{\theta}$ in terms of its coordinates in a specific reference system. We will use a reference system based on the orthogonal vectors $\{\mathbf{u}, \mathbf{n}, \mathbf{u} \times \mathbf{n}\}$, where $\mathbf{u}$ is the unit vector of the finite screw axis (FSA), and $d \mathbf{q}$ is contained in the $\{\mathbf{u}, \mathbf{n}\}$ plane:

$$
d \mathbf{q}=d q_{u} \mathbf{u}+d q_{n} \mathbf{n}
$$

The relation between the rotation artifact $d \mathbf{q}$ and its effect on the measured RV, $d \boldsymbol{\theta}$, can be expressed by the following formulae (Appendix I):

Error in $\boldsymbol{\theta}$ modulus: $\quad d \theta=d q_{u}$ 
Error in $\boldsymbol{\theta}$ orientation: $\quad d \alpha=|d \mathbf{u}|=\frac{d q_{n}}{2 \sin (\theta / 2)}$

Errors in $\boldsymbol{\theta}$ components:

$$
\begin{aligned}
& d \theta_{u}=d \theta=d q_{u} \\
& d \theta_{n}=\frac{\theta d q_{n}}{2 \tan (\theta / 2)} \\
& d \theta_{u \times n}=\left(\frac{\theta}{2}\right) d q_{n}
\end{aligned}
$$

Equations (4) and (5) show that the error in the RV module is equal to the component of $d \mathbf{q}$ along the FSA, whereas its normal component causes a deviation of the FSA, that also depends on $\theta$. The well-known issue of FSA indetermination for small angles is reflected by the term $\sin (\theta / 2)$ in the denominator of Eq. (5).

Equations (6) through (8) show how $d \mathbf{q}$ is transmitted to the components of $d \boldsymbol{\theta}$. The component along the FSA is transmitted as is. On the other hand, the error in the normal direction is scaled by the factor $\theta /[2 \tan (\theta / 2)]$, whose maximum value is near 1 for small angles, and decreases as the angle grows larger. Besides, there is an "intruder" component in the common normal to $d \mathbf{q}$ and $d \boldsymbol{\theta}$, whose value is $d q_{n}$ scaled by the half angle in radians.

All in all, when rotations are represented by $\mathrm{RV}$, the size of the rotation error keeps bounded, although orientation errors cannot be prevented due to the ill-determination of the FSA.

\section{Transmission errors to Euler angles}

Let $\mathbf{R}$ be the rotation matrix associated to the bone motion:

$$
\mathbf{R}=\mathbf{R}_{1}\left(\Phi_{1}\right) \mathbf{R}_{2}\left(\Phi_{2}\right) \mathbf{R}_{3}\left(\Phi_{3}\right)
$$


where $\left[\Phi_{1}, \Phi_{2}, \Phi_{3}\right]$ are the EA of the chosen sequence, and $\mathbf{R}_{1}, \mathbf{R}_{2}, \mathbf{R}_{3}$ are their corresponding rotation matrices. The axes of rotation are given in the sequential positions of a frame attached to the bone by the unit vectors $\left\{\mathbf{u}_{1}, \mathbf{u}_{2}, \mathbf{u}_{3}\right\}$.

The observed EA corresponding to the movement of the markers are $\left[\Phi_{M C 1}, \Phi_{M C 2}, \Phi_{M C 3}\right]$, which have an error $\left[d \Phi_{1}, d \Phi_{2}, d \Phi_{3}\right]$ with respect to the "true", free of STA, angles:

$$
d \Phi_{1}=\Phi_{M C 1}-\Phi_{1} ; d \Phi_{2}=\Phi_{M C 2}-\Phi_{2} ; d \Phi_{3}=\Phi_{M C 3}-\Phi_{3}
$$

By assuming $\mathrm{d} \theta$ to be small $\left(\mathrm{d} \theta<10^{\circ}\right)$, it is possible to prove that (Appendix II):

$$
d \mathbf{q}=d \Phi_{1} \mathbf{R}_{3}^{T} \mathbf{R}_{2}^{T} \mathbf{u}_{1}+d \Phi_{2} \mathbf{R}_{3}^{T} \mathbf{u}_{2}+d \Phi_{3} \mathbf{u}_{3}
$$

Table 1. Propagation of STA related rotation of the marker cluster, $d \mathbf{q}$, to Euler angles obtained by solving eq. (11) for the most-used sequences in human movement analysis. In order to simplify the writing of equations we denote $\cos \Phi_{\mathrm{i}}$ by ci and $\sin \Phi_{\mathrm{j}}$ by sj.

\begin{tabular}{|l|l|c|c|c|}
\hline Sequence & \multicolumn{1}{|c|}{ Joint } & \multicolumn{1}{c|}{$\mathbf{d \Phi _ { 1 }}$} & $\mathbf{d} \Phi_{\mathbf{2}}$ & $\mathbf{d} \boldsymbol{\Phi}_{\mathbf{3}}$ \\
\hline XY'Z”, & Knee [13] & $\frac{c 3 d q_{x}-s 3 d q_{y}}{c 2}$ & $s 3 d q_{x}+c 3 d q_{y}$ & $d q_{z}-\frac{s 2}{c 2}\left(c 3 d q_{x}-s 3 d q_{y}\right)$ \\
\hline $\begin{array}{l}\text { Ankle [14] } \\
\text { Hip [14] } \\
\text { Spine [14] } \\
\text { Thorax [15] } \\
\text { Elbow [15] } \\
\text { Humero-ulnar [15] } \\
\text { Hand/wrist [15] }\end{array}$ & $\frac{c 3 d q_{z}-s 3 d q_{x}}{c 2}$ & $s 3 d q_{z}+c 3 d q_{x}$ & $d q_{y}-\frac{s 2}{c 2}\left(c 3 d q_{z}-s 3 d q_{x}\right)$ \\
\hline YX'Y”, & $\begin{array}{l}\text { Gleno-humeral [15] } \\
\text { Thorax-humerus [15] }\end{array}$ & $\frac{s 3 d q_{x}-c 3 d q_{z}}{s 2}$ & $c 3 d q_{x}+s 3 d q_{z}$ & $d q_{y}-\frac{c 2}{s 2}\left(s 3 d q_{x}-c 3 d q_{z}\right)$ \\
\hline
\end{tabular}

The solution to this system of equations depends on the chosen squence. Table 1 shows the solutions for the most-used Euler sequences in the analysis of human 
movements. As can be seen in these equations, the components of $d \mathbf{q}$ have strong, nonlinear crossed effects on EA. The error of the first and third angles, $d \Phi_{1}, d \Phi_{3}$, is very sensitive to the value of the second angle $\Phi_{2}$, such that when it is near a singular configuration (small values of $\cos \Phi_{2}$ or $\sin \Phi_{2}$, depending on the type of sequence), the error experiments an important amplification, and may be quite larger than the rotation artifact that creates them. The sine and cosine of the third angle $\Phi_{3}$ have crossed scaling effects on the components of $d \mathbf{q}$. On the other hand, the value of the first rotation angle $\Phi_{1}$ does not affect the rotation error.

\section{Validation}

\section{Experimental}

An experiment was performed to test the proposed model of error propagation, using a mechanical analogue to a "ball-and-socket" joint, like the gleno-humeral or the hip joint. It consisted in a metallic rod with a spherical joint in one end, covered by a flexible foam loosely attached to the bar and stuck to the fixed base (Fig. 2). A rigid plate with 20 reflective markers was attached to the free end of the bar, to have an artifact-free gold standard. A $3 \times 4$ marker grid $(100 \times 166 \mathrm{~mm})$ was fixed to the foam surface. The motion of this mechanism was recorded by 4 cameras (Pulnix), at 100 frames per second, calibrated by bundle adjustment. The position error of the markers was $0.3 \mathrm{~mm}$, and their trajectories were smoothed by the procedure described in [17]. The upper bound of the angular instrumental errors was estimated from the errors in markers location as described in [18], obtaining a maximum angular error of $0.2^{\circ}$. 


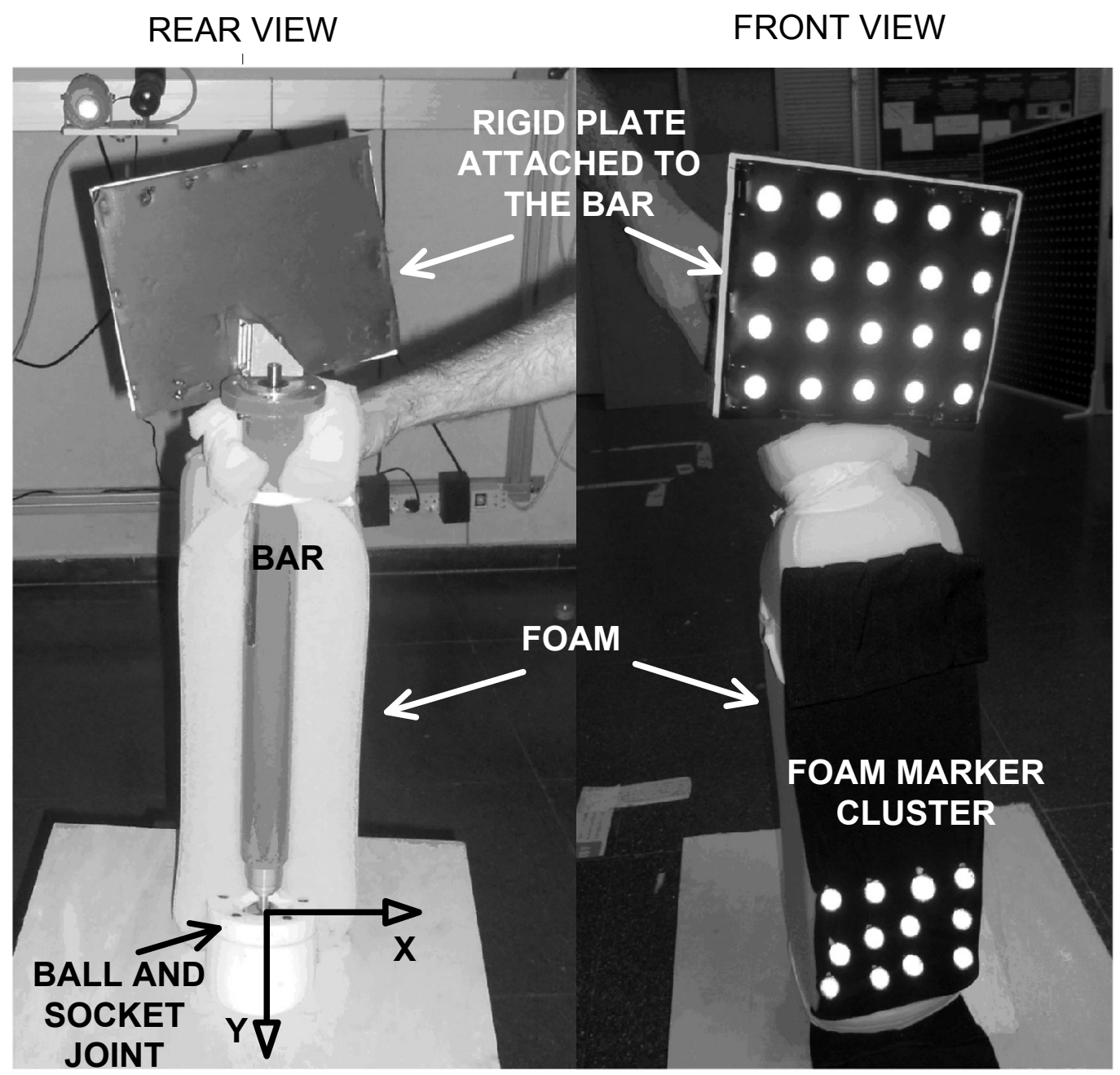

Figure 2. Experimental device to simulate a deformable marker set that moves relative to the bone.

All motions were recorded in a fixed reference system, whose coordinate axes coindiced with the standard anatomical systems in the reference position (Fig.1). In this position, the bar was held vertically, and then it was cyclically rotated in an alternating half circumduction movement with a $30^{\circ}$ tilt for 5 seconds.

The following variables were calculated from marker positions:

a) Rotation of the bar, as described in [8]. The result was expressed in the fixed frame as the RV, $\boldsymbol{\theta}$, and the EA sequences $\left[\Phi_{1}, \Phi_{2}, \Phi_{3}\right]$ $\left(X Y^{\prime} Z^{\prime \prime}, Z X^{\prime} Y^{\prime \prime}\right.$, and $\left.Y X^{\prime} Y^{\prime \prime}\right)$. 
b) Rotation of the foam marker cluster with respect to the fixed base $\left(\boldsymbol{\theta}_{M C}\right.$ or $\left.\left[\Phi_{M C 1}, \Phi_{M C 2}, \Phi_{M C 3}\right]\right)$

c) Infinitesimal approximation to the artifact, $d \mathbf{q}$, calculated as described in [16].

d) Estimated rotation of the marker cluster $\left(\boldsymbol{\theta}_{M C e}\right.$ or $\left.\left[\Phi_{M C e 1}, \Phi_{M C e 2}, \Phi_{M C e 3}\right]\right)$, obtained from (a) and (c) by adding the infinitesimal rotation $d \mathbf{q}$ and the finite rotation $\boldsymbol{\theta}$.

e) Measured STA errors derived from the difference between (a) and (b): $d \boldsymbol{\theta}_{m}=\boldsymbol{\theta}_{M C}-\boldsymbol{\theta}, d \Phi_{m i}=\Phi_{M C i}-\Phi_{i}$.

f) Estimated STA errors from the difference between (a) and (d): $d \boldsymbol{\theta}_{e}=\boldsymbol{\theta}_{M C e}-\boldsymbol{\theta}$, $d \Phi_{e i}=\Phi_{M C e i}-\Phi_{i}$

Then, the following comparisons were done:

- Measured and estimated marker cluster rotations, (b) vs. (d). This comparison was done to constrast the assumed hypothesis, that an infinitesimal rotation can account for the difference between the motion of the bone and the observer marker cluster.

- Measured and estimated errors due to STA, (e) vs. (f), represented by the maximum difference. This comparison was done to validate the error transmission model.

- STA rotation coordinates $d \mathbf{q}$ vs. rotation errors represented as RV or EA, for each type of representation.

\section{Results}

Figure 3 shows the measured rotations of both bar and foam, expressed as the RV and the three EA sequences. The ranges of motion depended on how rotations were represented. Using RV coordinates, they were $24^{\circ}$ in the $\mathrm{X}$ - and $\mathrm{Y}$-axes, and $55^{\circ}$ in the 
Z-axis. EA had similar values (with differences lower than $7^{\circ}$ ) for the $X Y^{\prime} Z^{\prime \prime}$ and $Z Y^{\prime} X^{\prime \prime}$ sequences, but they were very different for the $Y X^{\prime} Y^{\prime \prime}$ sequence.

Figure 4 shows the components of the calculated rotation artifact $d \mathbf{q}$, whose average value was $\left[0.6^{\circ}, 1.5^{\circ}, 0.6^{\circ}\right]$ and whose peak absolute value was around $6^{\circ}$, for the axial rotation coordinate $(Z)$.
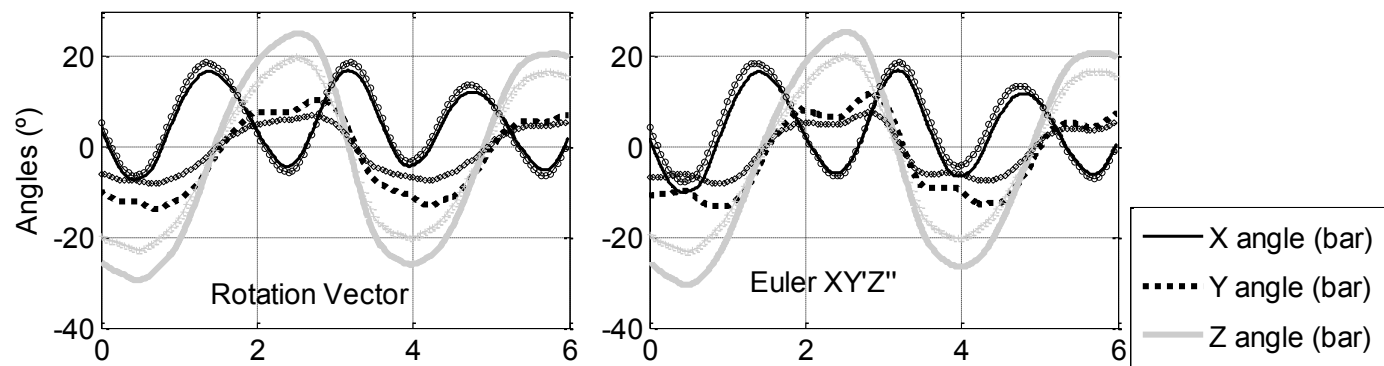

-...Y angle (bar)

Z angle (bar)
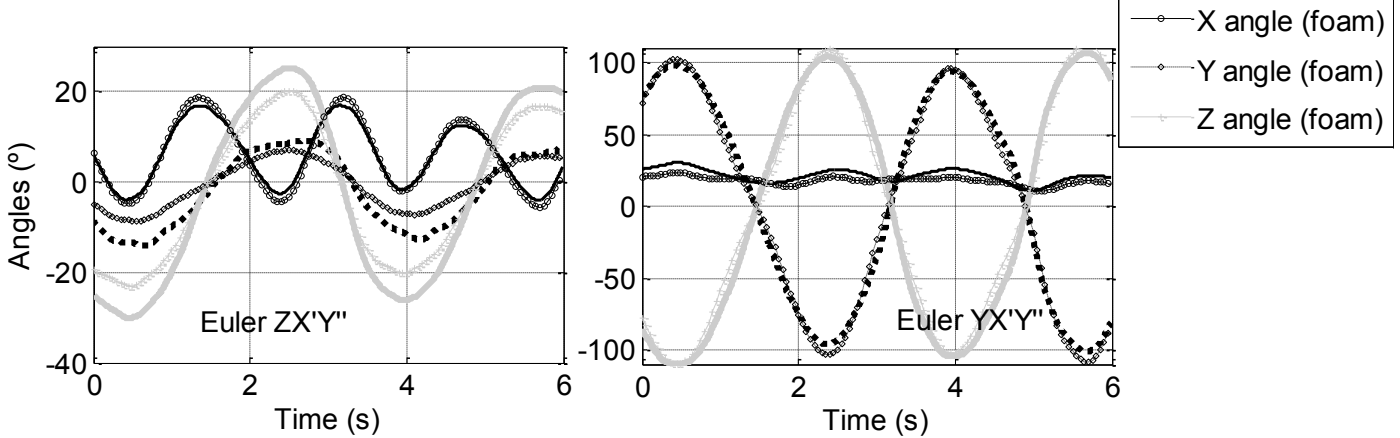

Figure 3. RV and EA components of the bar and foam observed rotations. For the $Y X$ 'Y" sequence, the curve labeled as " $Z$ " corresponds to the third angle ( $Y$ "). 


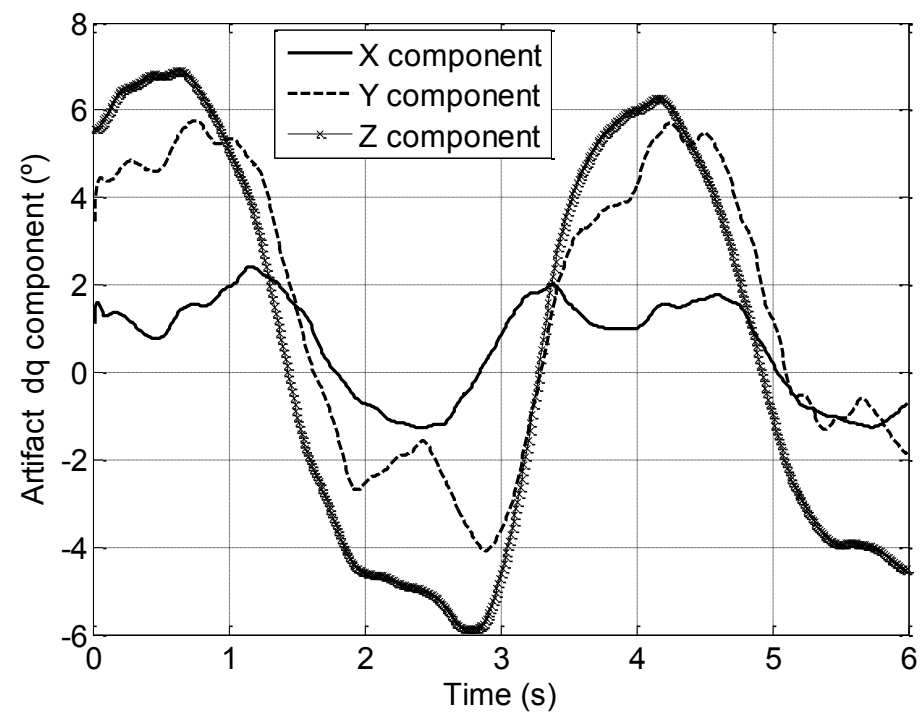

Figure 4. Components of the rotation artifact $d \mathbf{q}$ (relative motion of the foam with respect to the bar). Note that $d \boldsymbol{q}$ has not zero values at $t=0$. This is because the reference position does not coincide with the initial position of the measured motion.

The estimated foam rotations $\left(\boldsymbol{\theta}_{M C e}\right.$ or $\left.\left[\Phi_{M C e 1}, \Phi_{M C e 2}, \Phi_{M C e 3}\right]\right)$, calculated by composing $d \mathbf{q}$ and $\boldsymbol{\theta}$, were virtually identical to the observed values ( $\boldsymbol{\theta}_{M C}$ or $\left.\left[\Phi_{M C 1}, \Phi_{M C 2}, \Phi_{M C 3}\right]\right)$, with differences lower to $0.07^{\circ}$ for any component an sequence.
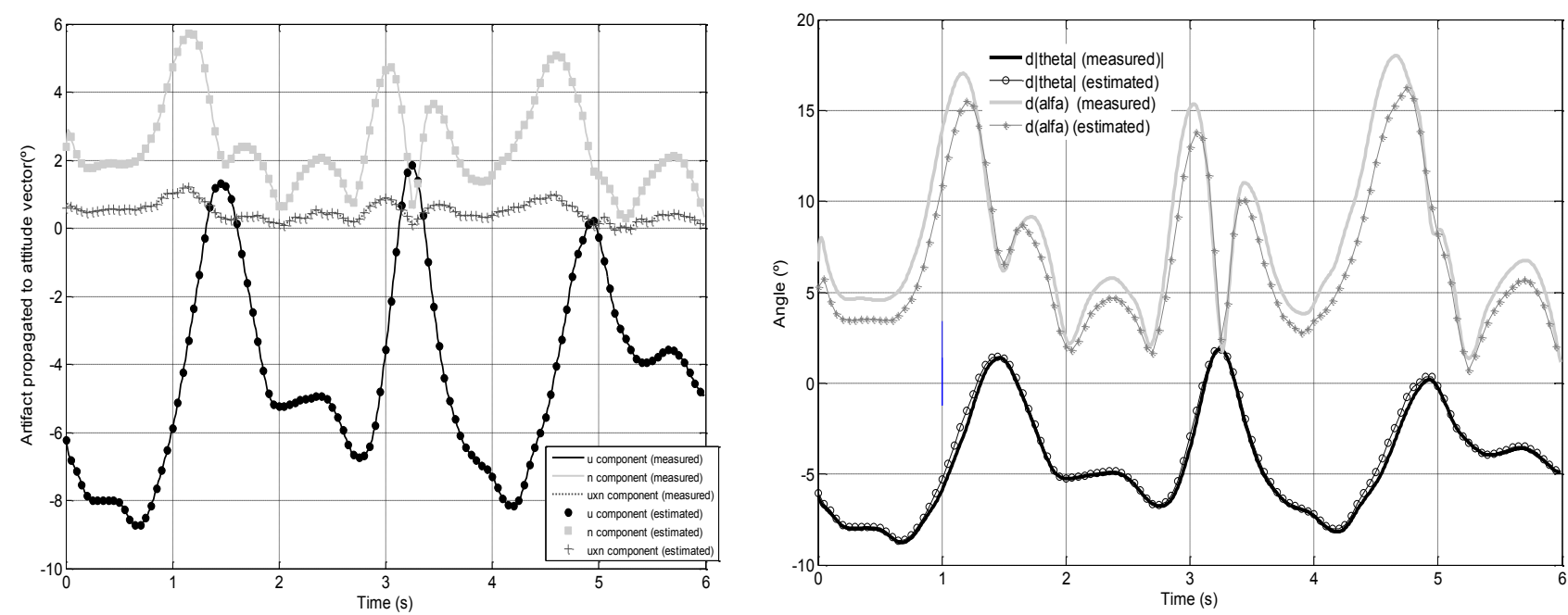

Figure 5. Estimated and measured RV errors. (a) Coordinates of $R V$ errors in the reference system $\{\mathbf{u}, \mathbf{n}, \mathbf{u} \times \mathbf{n}\}$. (b) Module and orientation error of the $R V$. 
Figure 5 shows the measured and estimated values of the RV error, expressed as their coordinates in the reference system $\{\mathbf{u}, \mathbf{n}, \mathbf{u} \times \mathbf{n}\}$, as well as the module and orientation errors. The greatest component was in the direction of the FSA $(\mathbf{u})$, and reached $8^{\circ}$ in absolute value. That was also the value of the RV module error; the orientation error had peak values over $15^{\circ}$. The difference between $d \boldsymbol{\theta}_{m}$ and $d \boldsymbol{\theta}_{m e}$ was lower than $0.2^{\circ}$ for all coordinates, and $0.9^{\circ}$ for its module. The difference between measured and estimated orientation of the RV was relatively greater (standard deviation $0.93^{\circ}$, maximum $\left.3.4^{\circ}\right)$.

Table 2 summarizes the differences between measured and estimated errors of RV and EA. Differences were again lower than $0.2^{\circ}$ for the RV, and lower than $0.4^{\circ}$ for the $X Y^{\prime} Z^{\prime \prime}$ and $Z Y^{\prime} X^{\prime \prime} E A$, but there were peak differences over $2^{\circ}$ for the $Y^{\prime} Y^{\prime \prime}$ sequence.

Table 2. Fit of the STA transmission model. Peak differences between measured STA errors $\left(d \boldsymbol{\theta}_{m}\right.$ or $\left.\left[d \Phi_{1}, d \Phi_{2}, d \Phi_{3}\right]\right)$ and estimated errors $\left(d \boldsymbol{\theta}_{e}\right.$ or $\left.\left[d \Phi_{e 1}, d \Phi_{e 2}, d \Phi_{e 3}\right]\right)$. EA errors are permutated so that they are ordered column-wise as $\mathrm{X}-\mathrm{Y}-\mathrm{Z}$ in all cases (in the YX'Y', sequence, $\mathrm{Z}$ stands for Y' ').

\begin{tabular}{|l|c|c|c|}
\hline & \multicolumn{2}{|c|}{ Max. difference between measured and } \\
& $\varepsilon_{X}$ & $\varepsilon_{Y}$ & $\varepsilon_{Z}$ \\
\hline Angle notation & 0.08 & 0.20 & 0.07 \\
\hline Rotation vector & 0.36 & 0.24 & 0.24 \\
\hline Euler XY'Z', & 0.07 & 0.36 & 0.23 \\
\hline Euler ZX'Y', & 2.19 & 0.50 & 2.16 \\
\hline Euler YX'Y', & & & \\
\hline
\end{tabular}


Figure 6 and Table 3 show the relation between the rotation artifact $d \mathbf{q}$ and the errors transmitted to the kinematic analysis for the different representations. The lowest difference with respect to $d \mathbf{q}$ was obtained by the RV (maximum difference lower than $1^{\circ}$ ). The order of magnitude of EA errors for $X Y^{\prime} Z^{\prime \prime}$ and $Z Y^{\prime} X^{\prime \prime}$ was the same as $d \mathbf{q}$. On the other hand, EA errors of the $\mathrm{YX}^{\prime} \mathrm{Y}^{\prime \prime}$ sequence were remarkable greater.

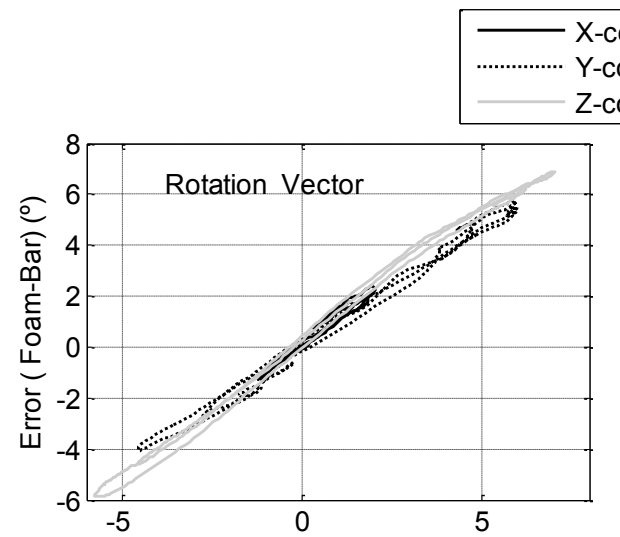

X-component

Y-component

-component
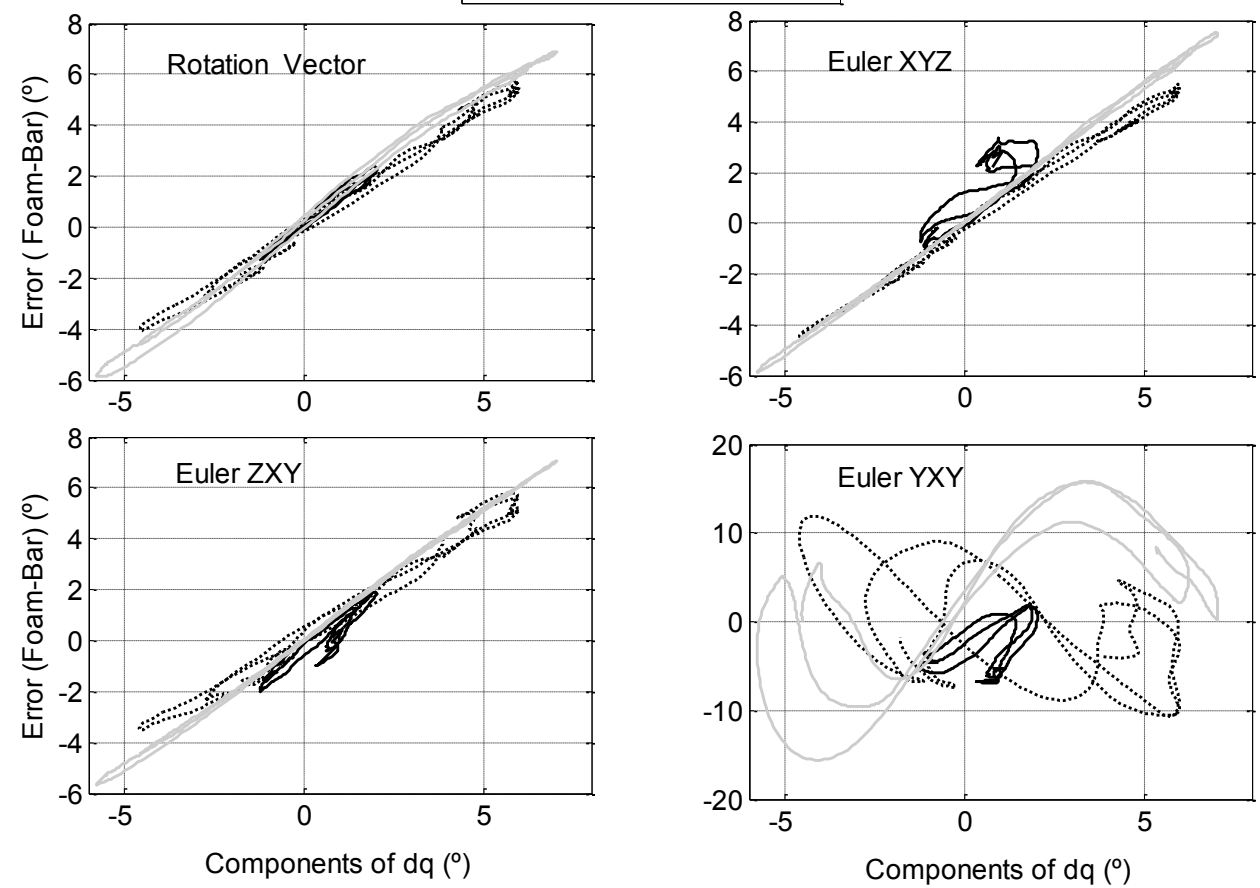

Figure 6. Components of the errors in $R V$ and $E A$ vs artifact dq . EA errors are permutated and related to the $X-Y-Z$ axes as in table 1. 
Table 3. Maximum differences between the components of $d \mathbf{q}$ and errors of RV and EA. EA errors are permutated and related to the X-Y-Z axes as in table 1.

\begin{tabular}{|l|c|c|c|}
\hline \multirow{2}{*}{ Angle notation } & \multicolumn{3}{|c|}{ Max $|\mathrm{d} \theta-\mathrm{dq}|(\mathrm{RV})$ or } \\
& \multicolumn{2}{|c|}{ Max $\mid \mathrm{d} \Phi$-dq (Euler sequences) } \\
\cline { 2 - 4 } & $\mathrm{X}\left(^{\circ}\right)$ & $\mathrm{Y}\left(^{\circ}\right)$ & $\mathrm{Z}\left(^{\circ}\right)$ \\
\hline Rotation vector & 0.623 & 0.758 & 0.855 \\
\hline Euler XY'Z'’ & 2.402 & 0.978 & 0.604 \\
\hline Euler ZX'Y'’ & 1.395 & 1.226 & 0.322 \\
\hline Euler YX'Y', & 7.895 & 16.373 & 12.571 \\
\hline
\end{tabular}

\section{Discussion and conclusion}

This work presents the equations that explain how STA errors are transmitted to rotation errors, for usual mathematical formalisms. To our knowledge, this is the first work that presents explict equations for predicting kinematic rotation errors as functions of the artifact, which is expressed as a rigid small rotation of the marker cluster with respect to the bone. The specific solutions of those equations have been derived for the RV and the EA sequences most frequently used in the field of biomechanics.

The resulting formulas show that the transmitted error depends on the specific formalism used to represent joint rotations. EA errors are affected by strong nonlinear relations with the underlying rotation artifact, which depend on the value of the "true" rotation in each instant, and may be intensely amplified near singular angle configurations. On the other hand, the relation between artifacts and RV errors is perfectly linear in the direction of the FSA and for the RV module, although its 
direction may be more affected due to nonlinear effects, specially when the rotation of the bone is small.

Similar relations were described by Woltring for accidental random errors and isotropic marker configurations [19]. This work extends such conclusions, with explicit formulas, for any type of errors and independently of the distribution of markers.

The theoretical formulas have been validated by an experiment with a deformable mechanical analogue, a frequent resource for validations of biomechanical models, since it allows an accurate control of error sources without invasive procedures $[20,21]$. The results confirm the predictions of the model. The greatest deviation occurred for the RV orientation error when rotations are small, and for the first and third EA in the YX'Y', sequence, for small elevation angles; this may be explained by a departure from the hypothesis of "small rotation artifact" in those cases. As expected by the formulas, RV errors were more similar to $d \mathbf{q}$ than EA errors, although there were remarkable differences between EA sequences. Nonlinear effects were less noticeable for $X Y^{\prime} Z^{\prime}$ and $Z Y^{\prime} X^{\prime \prime}$ (typically used for hip and knee joints) because the second rotation, was small, as normally occurs in human movements $\left(\cos \Phi_{2} \gg 0\right)$. On the other hand, there were great errors and departures from the model for $Y^{\prime} Y^{\prime \prime}$ (recommended for the gleno-humeral joint), since for that sequence the denominator was $\sin \Phi_{2}$, which approached zero in some instants - as when the arms are near the trunk.

The motion that was measured in the experiment may not correspond to the actual motion of a human limb, so the specific errors that were obtained cannot be taken as a model of the errors that occur in human motion analysis, although the order of magnitude of STA-related errors, measured between $5^{\circ}$ and $10^{\circ}$, is within the range of 
in-vivo experiments [2], and substantially greater than the estimated instrumental errors $\left(0.2^{\circ}\right)$. In vivol studies are also affected by other sources of error, like the definition of anatomical reference frames. This is absent in the reported analysis, which used a fixed, global reference frame, but in vivo studies may show up to $10^{\circ}$ of additional error due to that source [22]. Therefore, this limitation does not affect the general conclusions, which may be extended to any type of movement and artifact. All in all, the results that were obtained suggest that the direct comparison of measured and "artifact-free" EA do not constitute a general adequate reference for describing STA, since the resulting error largely depends on the actual rotation of the joint, as much or more than the actual artifact. Comparisons based on the RV are more natural, nearly linear, and less affected by the amplification of errors, which are always bounded.

\section{Acknowledgements}

This work has been funded by the Spanish Government and co-financed by EU FEDER funds (Grants DPI2009-13830-C02-01, DPI2009-13830-C02-02).

\section{Appendix I. Propagation of artifact $d \mathbf{q}$ to RV}

The relation between $d \boldsymbol{\theta}$ and the rotation artifact $d \mathbf{q}$ can indirectly be derived from the more straightforward relation between differential rotations and their effect on the Rodrigues vector [8]. Since $d \mathbf{q}$ is assummed to be small, the Rodrigues vector associated to this differential rotation is $d \mathbf{q} / 2$, and the observed rotation, $\boldsymbol{\Omega}_{M C}$, can be obtained by adding the bone rotation $\Omega$ to it, i.e.:

$$
\boldsymbol{\Omega}_{M C}=\boldsymbol{\Omega}+d \boldsymbol{\Omega}=\frac{(d \mathbf{q} / 2)+\boldsymbol{\Omega}+\boldsymbol{\Omega} \times d \mathbf{q} / 2}{1-\boldsymbol{\Omega} \cdot d \mathbf{q} / 2}
$$


Neglecting second-order differential terms, this expression can be simplified as:

$$
d \mathbf{\Omega} \approx \frac{1}{2}[d \mathbf{q}+\mathbf{\Omega} \times d \mathbf{q}+(\mathbf{\Omega} \cdot d \mathbf{q}) \mathbf{\Omega}]
$$

Now, the definition of $\boldsymbol{\Omega}$ w.r.t. $\boldsymbol{\theta}$ can be differentiated to give the relation between $d \boldsymbol{\Omega}$ and the error in module and direction of $\boldsymbol{\theta}$ :

$$
\mathbf{\Omega}=\tan \left(\frac{\theta}{2}\right) \mathbf{u} \Rightarrow d \mathbf{\Omega}=\frac{1}{2}\left(1+\Omega^{2}\right) d \theta \mathbf{u}+\Omega d \mathbf{u}
$$

The relation between the error of the RV and $d \mathbf{q}$ is thus obtained by equating the right hand sides of Eqs. (I2) and (I3). This relation can be made more explicit, by defining $d \mathbf{q}$ in a reference system based on the orthogonal vectors $\{\mathbf{u}, \mathbf{n}, \mathbf{u} \times \mathbf{n}\}$, where $\mathbf{u}$ is the unit vector of the finite screw axis (FSA), and $d \mathbf{q}$ is contained in the $\{\mathbf{u}, \mathbf{n}\}$ plane:

$$
d \mathbf{q}=d q_{u} \mathbf{u}+d q_{n} \mathbf{n}
$$

Substituting this definition of $d \mathbf{q}$ in (I3):

$$
d \mathbf{\Omega}=\frac{1}{2}\left(1+\Omega^{2}\right) d q_{u} \mathbf{u}+\frac{1}{2}\left(d q_{n} \mathbf{n}+\Omega d q_{n}(\mathbf{u} \times \mathbf{n})\right)
$$

Now, equating the orthogonal terms of Eqs.(I3) and (I5):

$$
\begin{aligned}
& d \theta=d q_{u} \\
& d \mathbf{u}=\frac{1}{2} d q_{n}\left(\frac{\mathbf{n}}{\Omega}+\mathbf{u} \times \mathbf{n}\right)
\end{aligned}
$$

And since the error in the direction of the RV is assumed to be small, its angle $d \alpha$ (in radians) is just the module of $d \mathbf{u}$ :

$$
d \alpha=|d \mathbf{u}|=\frac{1}{2} d q_{n}\left(\frac{1+\Omega^{2}}{\Omega^{2}}\right)^{1 / 2}=\frac{d q_{n}}{2 \sin (\theta / 2)}
$$

The value of $d \boldsymbol{\theta}$ can also be represented by its coordinates in the above mentioned reference system:

$$
d \boldsymbol{\theta}=d \theta_{u} \mathbf{u}+d \theta_{n} \mathbf{n}+d \theta_{u \times n} \mathbf{u} \times \mathbf{n}
$$


Comparing the terms of this definition with Eqs. (2) and (I6-I7):

$$
\begin{aligned}
& d \theta_{u}=d \theta=d q_{u} \\
& d \theta_{n}=\frac{\theta d q_{n}}{2 \tan (\theta / 2)} \\
& d \theta_{u \times n}=\left(\frac{\theta}{2}\right) d q_{n}
\end{aligned}
$$

\section{Appendix II. Proof of Equation (11)}

Let $\mathbf{R}$ be the rotation matrix associated to the bone motion:

$$
\mathbf{R}=\mathbf{R}_{1}\left(\Phi_{1}\right) \mathbf{R}_{2}\left(\Phi_{2}\right) \mathbf{R}_{3}\left(\Phi_{3}\right)
$$

where $\left[\Phi_{1}, \Phi_{2}, \Phi_{3}\right]$ are the Euler angles of the chosen sequence, and $\mathbf{R}_{1}, \mathbf{R}_{2}, \mathbf{R}_{3}$ are their corresponding rotation matrices. The axes of rotation are given in the sequential positions of a frame attached to bone B by the unit vectors $\left\{\mathbf{u}_{1}, \mathbf{u}_{2}, \mathbf{u}_{3}\right\}$ (e.g. $\{\mathbf{i}, \mathbf{k}, \mathbf{j}\}$ for the $X Z^{\prime} Y^{\prime \prime}$ 'sequence, $\{\mathbf{j}, \mathbf{i}, \mathbf{j}\}$ for $Y X^{\prime} Y^{\prime}$, , etc.), which expressed in a fixed frame as:

$$
\mathbf{e}_{1}=\mathbf{R}_{1} \mathbf{u}_{1} ; \mathbf{e}_{2}=\mathbf{R}_{1} \mathbf{R}_{2} \mathbf{u}_{2} ; \mathbf{e}_{3}=\mathbf{R}_{1} \mathbf{R}_{2} \mathbf{R}_{3} \mathbf{u}_{3}
$$

The observed Euler angles corresponding to the movement of the maker cluster are $\left[\Phi_{M C 1}, \Phi_{M C 2}, \Phi_{M C 3}\right]$, which have an error $\left[d \Phi_{1}, d \Phi_{2}, d \Phi_{3}\right]$ with respect to the "true" angles:

$$
d \Phi_{1}=\Phi_{C 1}-\Phi_{1} ; d \Phi_{2}=\Phi_{C 2}-\Phi_{2} ; d \Phi_{3}=\Phi_{C 3}-\Phi_{3}
$$

The angles $\left[\Phi_{M C 1}, \Phi_{M C 2}, \Phi_{M C 3}\right]$ are the same as we would obtain if we composed the rotation defined by $\left[\Phi_{1}, \Phi_{2}, \Phi_{3}\right]$ and then the STA rotation $d \mathbf{q}$. Note that $d \mathbf{q}$ is thec rotation associated to the STAt at time $t$, but expressed in the moving, bone-embedded frame. In the fixed frame (position $\mathrm{B}_{0}$ and $\mathrm{MC}_{2}$ in Fig. 1) the STA rotation $d \mathbf{q}$ is expressed as ${ }^{0} d \mathbf{q}$ :

$$
{ }^{0} d \mathbf{q}=\mathbf{R} d \mathbf{q}=\mathbf{R}_{1} \mathbf{R}_{2} \mathbf{R}_{3} d \mathbf{q}
$$

If the errors in the Euler angles are small, it is possible to calculate an additive composition of the differential angles in the fixed frame. The result will be the same as the STA rotation expressed in this frame, ${ }^{0} d \mathbf{q}$ : 


$$
{ }^{0} d \mathbf{q}=d \Phi_{1} \mathbf{e}_{1}+d \Phi_{2} \mathbf{e}_{2}+d \Phi_{3} \mathbf{e}_{3}
$$

Combining Eqs (II2), (II4) and (II5) we arrive at the following relation between $d \mathbf{q}$ and the error in Euler angles:

$$
d \mathbf{q}=d \Phi_{1} \mathbf{R}_{3}^{T} \mathbf{R}_{2}^{T} \mathbf{u}_{1}+d \Phi_{2} \mathbf{R}_{3}^{T} \mathbf{u}_{2}+d \Phi_{3} \mathbf{u}_{3}
$$




\section{References}

[1] Leardini A., Chiari L., Croce U. D., and Cappozzo A., 2005, "Human movement analysis using stereophotogrammetry," Gait Posture, 21(2), pp. 212-225.

[2] Peters A., Galna B., Sangeux M., Morris M., and Baker R., 2010, "Quantification of soft tissue artifact in lower limb human motion analysis: A systematic review," Gait Posture, 31(1), pp. 1-8.

[3] Reinschmidt C., van den Bogert A. ., Lundberg A., Nigg B. ., Murphy N., Stacoff A., and Stano A., 1997, "Tibiofemoral and tibiocalcaneal motion during walking: external vs. skeletal markers," Gait Posture, 6(2), pp. 98-109.

[4] Lucchetti L., Cappozzo A., Cappello A., and Croce U. D., 1998, "Skin movement artefact assessment and compensation in the estimation of knee-joint kinematics," J. Biomech., 31(11), pp. 977-984.

[5] Benoit D. L., Ramsey D. K., Lamontagne M., Xu L., Wretenberg P., and Renström P., 2006, "Effect of skin movement artifact on knee kinematics during gait and cutting motions measured in vivo," Gait Posture, 24(2), pp. 152-164.

[6] Nester C., Jones R. K., Liu A., Howard D., Lundberg A., Arndt A., Lundgren P., Stacoff A., and Wolf P., 2007, "Foot kinematics during walking measured using bone and surface mounted markers," J. Biomech., 40(15), pp. 3412-3423.

[7] Andersen M. S., Benoit D. L., Damsgaard M., Ramsey D. K., and Rasmussen J., 2010, "Do kinematic models reduce the effects of soft tissue artefacts in skin marker-based motion analysis? An in vivo study of knee kinematics," J. Biomech., 43(2), pp. 268-273.

[8] Page Á., de Rosario H., Mata V., and Atienza C., 2009, "Experimental Analysis of Rigid Body Motion. A Vector Method to Determine Finite and Infinitesimal Displacements From Point Coordinates," J. Mech. Des., 131(3), pp. 031005031005.

[9] Diebel J., 2006, Representing attitude: Euler angles, unit quaternions, and rotation vectors, Stanford University, Stanford, CA.

[10] Woltring H. J., 1994, "3-D attitude representation of human joints: A standardization proposal,” J. Biomech., 27(12), pp. 1399-1414.

[11] Choi A. R., Rim Y. H., Kim Y. S., and Mun J. H., 2005, "A position based kinematic method for the analysis of human gait," J. Mech. Sci. Technol., 19(10), pp. 1919-1931.

[12] Angeles J., 2007, Fundamentals of Robotic Mechanical Systems: Theory, Methods, and Algorithms, Springer.

[13] Grood E. S., and Suntay W. J., 1983, “A joint coordinate system for the clinical description of three-dimensional motions: application to the knee," J. Biomech. Eng., 105(2), pp. 136-144.

[14] Wu G., Siegler S., Allard P., Kirtley C., Leardini A., Rosenbaum D., Whittle M., D’Lima D. D., Cristofolini L., Witte H., Schmid O., and Stokes I., 2002, "ISB recommendation on definitions of joint coordinate system of various joints for the reporting of human joint motion - part I: ankle, hip, and spine," J. Biomech., 35(4), pp. 543-548.

[15] Wu G., van der Helm F. C. T., (DirkJan) Veeger H. E. J., Makhsous M., Van Roy P., Anglin C., Nagels J., Karduna A. R., McQuade K., Wang X., Werner F. W., and Buchholz B., 2005, "ISB recommendation on definitions of joint 
coordinate systems of various joints for the reporting of human joint motion - Part II: shoulder, elbow, wrist and hand," J. Biomech., 38(5), pp. 981-992.

[16] Rosario H. de, Page A., Besa A., Mata V., and Conejero E., 2012, "Kinematic description of soft tissue artifacts: quantifying rigid versus deformation components and their relation with bone motion," Med. Biol. Eng. Comput., 50(11), pp. 1173-1181.

[17] Page A., Candelas P., and Belmar F., 2006, "On the use of local fitting techniques for the analysis of physical dynamic systems," Eur. J. Phys., 27(2), p. 273.

[18] Page A., Rosario H. D., Mata V., Hoyos J. V., and Porcar R., 2006, "Effect of marker cluster design on the accuracy of human movement analysis using stereophotogrammetry," Med. Biol. Eng. Comput., 44(12), pp. 1113-1119.

[19] Woltring H. J., Long K., Osterbauer P. J., and Fuhr A. W., 1994, "Instantaneous helical axis estimation from 3-D video data in neck kinematics for whiplash diagnostics," J. Biomech., 27(12), pp. 1415-1432.

[20] Camomilla V., Cereatti A., Vannozzi G., and Cappozzo A., 2006, “An optimized protocol for hip joint centre determination using the functional method," J. Biomech., 39(6), pp. 1096-1106.

[21] MacWilliams B. A., 2008, "A comparison of four functional methods to determine centers and axes of rotations," Gait Posture, 28(4), pp. 673-679.

[22] Della Croce U., Leardini A., Chiari L., and Cappozzo A., 2005, "Human movement analysis using stereophotogrammetry: Part 4: assessment of anatomical landmark misplacement and its effects on joint kinematics," Gait Posture, 21(2), pp. 226-237. 